\title{
Modular forms of finite modular subgroups from magnetized D-brane models
}

\author{
Tatsuo Kobayashi and Shio Tamba \\ Department of Physics, Hokkaido University, Sapporo 060-0810, Japan
}

(Received 5 December 2018; published 6 February 2019)

\begin{abstract}
We study modular transformation of holomorphic Yukawa couplings in magnetized D-brane models. It is found that their products are modular forms, which are nontrivial representations of finite modular subgroups, e.g., $S_{3}, S_{4}, \Delta(96)$ and $\Delta(384)$.

DOI: 10.1103/PhysRevD.99.046001
\end{abstract}

\section{INTRODUCTION}

The origin of the flavor structure in the quark and lepton sectors is one of the unsolved but important mysteries in particle physics. The quark and lepton masses have a hierarchy. The lepton mixing angles are large, while the quark mixing angles are small. Many studies have been done in order to understand this flavor structure. Among them, non-Abelian discrete flavor symmetries are one of the most interesting approaches. Indeed, many types of model building have been studied by use of various nonAbelian discrete groups such as $S_{N}, A_{N}, D_{N}, \Delta\left(3 N^{2}\right)$, $\Delta\left(6 N^{2}\right)$, etc. (See for review [1-3].)

Superstring theory is a promising candidate for a unified theory of all the interactions including gravity and matter particles such as quarks and leptons as well as the Higgs particle. The four-dimensional (4D) low-energy effective field theory of superstring theory has several symmetries. In particular, some non-Abelian discrete flavor symmetries appear in the 4D effective field theory from superstring theory within certain compactifications. That is, heterotic string theory on orbifolds leads to non-Abelian discrete flavor symmetries, e.g., $D_{4}$, and $\Delta(54)$ [4]. (See also Refs. [5-8].) ${ }^{1}$ In addition, magnetized D-brane models as well as intersecting D-brane models in type II superstring theory can realize similar discrete flavor symmetries [10-14].

In addition, torus and orbifold compactifications have the so-called modular symmetry. Recently, in Ref. [15,16], the modular transformation behavior of zero-modes was studied in magnetized D-brane models. (See also Ref. [17].) Such behavior was also studied in

\footnotetext{
${ }^{1}$ In Ref. [9], a relation between gauge symmetries and nonAbelian flavor symmetries is discussed at the enhancement point.

Published by the American Physical Society under the terms of the Creative Commons Attribution 4.0 International license. Further distribution of this work must maintain attribution to the author(s) and the published article's title, journal citation, and DOI. Funded by SCOAP ${ }^{3}$.
}

heterotic orbifold models [18-20]. These modular transformations act nontrivially on zero-modes. In this sense, the modular symmetry is a sort of flavor symmetry. However, the modular symmetry also transforms Yukawa couplings as well as higher order couplings, while these couplings are trivial singlets under the conventional flavor symmetries.

One interesting aspect is that the modular group $\Gamma$ includes finite subgroups such as $S_{3}, A_{4}, S_{4}$ and $A_{5}$, which have been used for flavor model building as mentioned above. (See e.g., Ref. [21].) Inspired by these aspects, recently a new approach to the lepton mass matrices was proposed in Ref. [22]. The three generations of leptons are assigned to non-trivial representations of the finite modular subgroup $A_{4}$. The couplings as well as neutrino masses are also assigned to modular forms, which are nontrivial representations under $A_{4}$. Such an idea was extended in Refs. [23-27] by use of other finite modular subgroups $S_{3}$ and $S_{4}$ in addition to $A_{4}$.

In these studies, the key ingredients are the modular forms of weight 2 , which are non-trivial representations of finite modular subgroups. ${ }^{2}$ Such modular forms are found for $S_{3}$ doublet, $A_{4}$ triplet, and $S_{4}$ doublet and triplet in Refs. [22-24]. Our purpose of this paper is to study a new type of constructions of modular forms for finite modular subgroups. We study Yukawa couplings in magnetized D-brane models. In particular, we study modular transformation of holomorphic Yukawa couplings, which are holomorphic functions of the modulus. Using them, we construct modular forms of modular weight 2 , which are nontrivial representations of finite modular subgroups.

This paper is organized as follows. In Sec. II, we review the modular symmetry in magnetized D-brane models and study modular symmetry of holomorphic Yukawa couplings. In Sec. III, we study products of holomorphic Yukawa couplings. We show that they correspond to

\footnotetext{
${ }^{2}$ See for modular forms, e.g., Refs. [28-30].
} 
modular forms of finite modular subgroups. Section IV is conclusion and discussion.

\section{MODULAR TRANSFORMATION IN MAGNETIZED D-BRANE MODELS}

In this section, we briefly review modular transformation of zero-mode wave functions in magnetized D-brane models. See for detail Refs. [15-17]. Then, we study modular transformation of holomorphic Yukawa couplings.

\section{A. Zero-mode wavefunction}

Here, we give a brief review on zero-mode wave functions on the two-dimensional torus $T^{2}$ with magnetic flux [17]. For simplicity, we concentrate on $T^{2}$ with $U(1)$ magnetic flux. We denote real coordinates by $x$ and $y$, and we also use the complex coordinate $z=x+\tau y$, where $\tau$ is the complex modular parameter. The metric on $T^{2}$ is given by

$$
g_{\alpha \beta}=\left(\begin{array}{ll}
g_{z z} & g_{z \bar{z}} \\
g_{\bar{z} z} & g_{\bar{z} \bar{z}}
\end{array}\right)=(2 \pi R)^{2}\left(\begin{array}{cc}
0 & \frac{1}{2} \\
\frac{1}{2} & 0
\end{array}\right) .
$$

In order to construct $T^{2}$, we identify $z \sim z+1$ and $z \sim z+\tau$.

We introduce the $U(1)$ magnetic flux $F$ on $T^{2}$,

$$
F=i \frac{\pi M}{\operatorname{Im} \tau}(d z \wedge d \bar{z})
$$

which corresponds to the vector potential,

$$
A(z)=\frac{\pi M}{\operatorname{Im} \tau} \operatorname{Im}(\bar{z} d z)
$$

Here we concentrate on vanishing Wilson lines.

We study the spinor field on $T^{2}$, which has two components,

$$
\Psi(z, \bar{z})=\left(\begin{array}{l}
\psi_{+} \\
\psi_{-}
\end{array}\right)
$$

On the above magnetic flux background, we solve the zeromode equation

$$
i D \Psi=0,
$$

for the spinor field with $U(1)$ charge $q$. The magnetic flux should be quantized by the Dirac condition such that $q M$ is integer. Either $\psi_{+}$or $\psi_{-}$has zero-modes exclusively for $q M \neq 0$. If $q M$ is positive, only $\psi_{+}$has $q M$ zero-modes, while $\psi_{-}$has no zero-mode. That is a chiral theory. Their zero-mode profiles are given by

$$
\psi^{j, q M}(z)=\mathcal{N} e^{i \pi q M z^{\frac{\operatorname{Im} z}{\operatorname{m} \tau}}} \cdot \vartheta\left[\begin{array}{c}
\frac{j}{q M} \\
0
\end{array}\right](q M z, q M \tau),
$$

with $j=0,1, \ldots,(q M-1)$, where $\vartheta$ denotes the Jacobi theta function,

$$
\vartheta\left[\begin{array}{l}
a \\
b
\end{array}\right](\nu, \tau)=\sum_{l \in \mathbf{Z}} e^{\pi i(a+l)^{2} \tau} e^{2 \pi i(a+l)(\nu+b)} .
$$

The normalization factor $\mathcal{N}$ is given by

$$
\mathcal{N}=\left(\frac{2 \operatorname{Im} \tau q M}{\mathcal{A}^{2}}\right)^{1 / 4},
$$

with $\mathcal{A}=4 \pi^{2} R^{2} \operatorname{Im} \tau$.

The ground states of scalar fields $\phi^{j, q M}$ also have the same profiles as $\psi^{j, q M}$. We can compute the Yukawa coupling including one scalar and two spinor fields, whose wave functions are written by $\phi^{i, M}, \psi^{j, N}$, and $\left(\psi^{k, M^{\prime}}\right)^{*}$, by carrying out the overlap integral of these wave fuctions. For simplicity, we have normalized the charges such as $q$ in a proper way and replaced $q M$ by $M$. Note that gauge invariance requires $M+N+M^{\prime}=0$. That is, $M^{\prime}$ must be negative if both $M$ and $N$ are positive. That is the reason why we consider the wave function $\left(\psi^{k, M^{\prime}}\right)^{*}$ [17]. Then, their Yukawa couplings are given by the wave function overlap integral [17],

$$
\begin{aligned}
Y_{i j k} & =g \int d^{2} z \phi^{i, M} \psi^{j, N}\left(\psi^{k, M^{\prime}}\right)^{*} \\
& =g\left(\frac{2 \operatorname{Im} \tau}{\mathcal{A}^{2}}\right)^{1 / 4} \sum_{m \in Z_{M^{\prime}}} \delta_{k, i+j+M m} \cdot \vartheta\left[\begin{array}{c}
\frac{N i-M j+M N m}{M N M^{\prime}} \\
0
\end{array}\right] \quad\left(0, M N M^{\prime} \tau\right),
\end{aligned}
$$

where $g$ is constant and $Z_{M^{\prime}}$ denotes the set of integers satisfying $0 \leq m<M^{\prime}$. Similarly, we can compute higher order couplings [31]. 


\section{B. Modular transformation}

Here, we study modular transformation. First we briefly review modular transformation of zero-modes $[15,16]$. (See also [17].)

The torus $T^{2}$ is constructed by $\mathbb{R}^{2} / \Lambda$, where the lattice $\Lambda$ is spanned by the vectors $\left(\alpha_{1}, \alpha_{2}\right)$, i.e., $\alpha_{1}=2 \pi R$ and $\alpha_{2}=2 \pi R \tau$. The same lattice is obtained by the following change of basis,

$$
\left(\begin{array}{c}
\alpha_{2}^{\prime} \\
\alpha_{1}^{\prime}
\end{array}\right)=\left(\begin{array}{ll}
a & b \\
c & d
\end{array}\right)\left(\begin{array}{l}
\alpha_{2} \\
\alpha_{1}
\end{array}\right)
$$

where $a, b, c, d$ are integer with satisfying $a d-b c=1$. That is an $S L(2, Z)$ transformation.

The modular parameter $\tau=\alpha_{2} / \alpha_{1}$ transforms as

$$
\tau \longrightarrow \gamma \tau=\frac{a \tau+b}{c \tau+d},
$$

under (10). This transformation includes two important generators, $S$ and $T$,

$$
\begin{gathered}
S: \tau \longrightarrow-\frac{1}{\tau}, \\
T: \tau \longrightarrow \tau+1 .
\end{gathered}
$$

They satisfy the following relations,

$$
S^{2}=1, \quad(S T)^{3}=1 .
$$

If we impose further algebraic relations in addition to Eq. (14), we can realize finite modular subgroups. For example, when we impose

$$
T^{N}=1,
$$

the modular subgroups are isomorphic to $S_{3}, A_{4}, S_{4}$ and $A_{5}$ for $N=2,3,4$, and 5, respectively. Also, we can obtain other finite modular subgroups by imposing further algebraic relations.

Following $[15,16]$, we restrict ourselves to even magnetic fluxes $M(M>0)$. Under $S$, the zero-mode wave functions transform as [15-17]

$$
\psi^{j, M} \rightarrow \frac{1}{\sqrt{M}} \sum_{k} e^{2 \pi i j k / M} \psi^{k, M} .
$$

On the other hand, the zero-mode wave functions transform as $[15,16]$

$$
\psi^{j, M} \rightarrow e^{\pi i j^{2} / M} \psi^{j, M},
$$

under $T$. Generically, the $T$-transformation satisfies

$$
T^{2 M} \psi^{j, M}=\psi^{j, M},
$$

on the zero-modes, $\psi^{j, M}$. Furthermore, in Ref. [15] it is shown that

$$
(S T)^{3} \psi^{j, M}=e^{\pi i / 4} \psi^{j, M},
$$

on the zero-modes, $\psi^{j, M}$ for generic case.

The holomorphic part of Yukawa couplings is given by

$$
X^{i, M}(\tau)=\vartheta\left[\begin{array}{c}
\frac{i}{M} \\
0
\end{array}\right] \quad(0, M \tau)
$$

We study modular transformations of $X^{i, M}(\tau)$. Hereafter, we often denote $X^{i, M}(\tau)$ by $X^{i, M}$. It is straightforward to study the modular transformation behavior of $X^{i, M}$ by using Eqs. (16) and (17). That is, the holomorphic function $X^{i, M}$ transforms as

$$
X^{j, M} \rightarrow \sqrt{\frac{-i \tau}{M}} \sum_{k} e^{2 \pi i j k / M} X^{k, M},
$$

under $S$, and

$$
X^{j, M} \rightarrow e^{\pi i j^{2} / M} X^{j, M},
$$

under $T$. Thus, the holomorphic Yukawa couplings as well as physical Yukawa couplings $Y_{i j k}$ transform nontrivially under the modular group. Note that the $T$ transformation is diagonal in this basis.

\section{MODULAR FORMS OF FINITE MODULAR SUBGROUPS}

Here, we study the construction of modular forms of weight 2 for finite modular subgroups. The modular forms of level $N$ and weight $w$ are holomorphic functions of the modulus $\tau$, which have the following modular transformation behavior:

$$
f_{i}(\gamma \tau)=(c \tau+d)^{w} \rho(\gamma)_{i j} f_{j}(\tau)
$$

where $\rho(\gamma)$ is a unitary representation of $\Gamma_{N}=\Gamma / \Gamma(N)$ with a principal congruence subgroup $\Gamma(N)$. The weights $w$ are even and modular forms of weight 2 are important, because other modular forms are obtained from their products.

Such modular forms of weight 2 are expected to be derived by productions of $X^{j, M}$ like $X^{i, M} X^{j, M} X^{k, M} X^{\ell, M}$ as seen from the behavior (21). In what follows, we study such products concretely. 


\section{A. $M=2$}

The $S$ and $T$ transformations are represented on $X^{j, 2}$ with $j=0,1$ by

$$
\left(\begin{array}{l}
X^{0,2} \\
X^{1,2}
\end{array}\right) \longrightarrow \sqrt{-\tau} \rho(S)\left(\begin{array}{l}
X^{0,2} \\
X^{1,2}
\end{array}\right), \quad \rho(S)=\sqrt{\frac{i}{2}}\left(\begin{array}{cc}
1 & 1 \\
1 & -1
\end{array}\right),
$$

and

$$
\left(\begin{array}{l}
X^{0,2} \\
X^{1,2}
\end{array}\right) \longrightarrow \rho(T)\left(\begin{array}{l}
X^{0,2} \\
X^{1,2}
\end{array}\right), \quad \rho(T)=\left(\begin{array}{ll}
1 & 0 \\
0 & i
\end{array}\right) .
$$

They satisfy the following algebraic relations,

$\rho(S)^{2}=i \mathbb{I}_{2 \times 2}, \quad \rho(T)^{4}=\mathbb{I}_{2 \times 2}, \quad(\rho(S) \rho(T))^{3}=-\mathbb{I}_{2 \times 2}$,

where $\mathbb{I}_{N \times N}$ denotes the $(N \times N)$ identity matrix. Similarly, representations of $S$ and $T$ on generic $X^{j, M}$ satisfy

$\rho(S)^{2}=i \mathbb{I}_{M \times M}, \quad \rho(T)^{2 M}=\mathbb{I}_{M \times M}, \quad(\rho(S) \rho(T))^{3}=-\mathbb{I}_{M \times M}$.

Now, let us study quartic tensor products of $X^{j, M}$ with $j=0,2$, i.e.,

$$
\begin{array}{lc}
\left(X^{0,2}\right)^{4}, & \left(X^{0,2}\right)^{3} X^{1,2}, \\
X^{0,2}\left(X^{1,2}\right)^{3}, & \left(X^{1,2}\right)^{4}
\end{array}
$$

These are five-dimensional representation of $S$ and $T$, which satisfy $\rho\left(S^{2}\right)=1, \rho\left((S T)^{3}\right)=1$, and $\rho\left(T^{4}\right)=1$. This five-dimensional representation is a reducible representation, and we can decompose it to two irreducible representations, a doublet and a triplet. The doublet corresponds to

$$
Z_{1}=\left(X^{0,2}\right)^{4}+\left(X^{1,2}\right)^{4}, \quad Z_{2}=2 \sqrt{3}\left(X^{0,2}\right)^{2}\left(X^{1,2}\right)^{2} .
$$

Then, on $\left(Z_{1}, Z_{2}\right)^{T}, S$ and $T$ are represented by

$$
\rho(S)=\frac{1}{2}\left(\begin{array}{cc}
-1 & -\sqrt{3} \\
-\sqrt{3} & 1
\end{array}\right), \quad \rho(T)=\left(\begin{array}{cc}
1 & 0 \\
0 & -1
\end{array}\right) .
$$

They satisfy $\quad \rho\left(S^{2}\right)=\mathbb{I}_{2 \times 2}, \quad \rho\left(T^{2}\right)=\mathbb{I}_{2 \times 2}, \quad$ and $\rho\left((S T)^{3}\right)=\mathbb{I}_{2 \times 2}$. That is nothing but $S_{3}$. Thus, $\left(Z_{1}, Z_{2}\right)$ are the modular forms corresponding to the $S_{3}$ doublet.

The other three elements are written by

$$
\begin{aligned}
& Z_{3}=\left(X^{0,2}\right)^{4}-\left(X^{1,2}\right)^{4}, \quad Z_{4}=2 \sqrt{2}\left(X^{0,2}\right)^{3} X^{1,2}, \\
& Z_{5}=2 \sqrt{2} X^{0,2}\left(X^{1,2}\right)^{3} .
\end{aligned}
$$

On $\left(Z_{3}, Z_{4}, Z_{5}\right)^{T}, S$ and $T$ are represented by

$$
\begin{aligned}
& \rho(S)=\frac{1}{2}\left(\begin{array}{ccc}
0 & -\sqrt{2} & -\sqrt{2} \\
-\sqrt{2} & -1 & 1 \\
-\sqrt{2} & 1 & -1
\end{array}\right), \\
& \rho(T)=\left(\begin{array}{ccc}
1 & 0 & 0 \\
0 & i & 0 \\
0 & 0 & -i
\end{array}\right) .
\end{aligned}
$$

They satisfy $\rho\left(S^{2}\right)=\mathbb{I}_{3 \times 3}, \rho\left(T^{4}\right)=\mathbb{I}_{3 \times 3}$, and $\rho\left((S T)^{3}\right)=$ $\mathbb{I}_{3 \times 3}$. That is isomorphic to $S_{4}$. Thus, $\left(Z_{3}, Z_{4}, Z_{5}\right)$ corresponds to the $S_{4}$ triplet.

\section{B. $M=4$}

Similarly, we can study the $M=4$ case. Note that $(S T)^{3}$ is a trivial transformation on $\tau$, but $(S T)^{3}$ transforms the lattice vectors $\left(\alpha_{1}, \alpha_{2}\right)$ to $\left(-\alpha_{1},-\alpha_{2}\right)$. The wave functions satisfy the following relation,

$$
\psi^{j, M}(-z)=\psi^{M-j, M}(z) .
$$

Thus, for $M>2$ it is convenient to use the following basis,

$$
\psi_{ \pm}^{j, M}=\frac{1}{\sqrt{2}}\left(\psi^{j, M}(z) \pm \psi^{M-j, M}(z)\right),
$$

except $j=0, M / 2$. in order to represent $S$. That is the $Z_{2}$ orbifold basis [32]. The $\psi_{+}^{j, M}$ are $Z_{2}$ even modes, while $\psi_{-}^{j, M}$ are $Z_{2}$ odd. The $\psi^{0, M}$ is always $Z_{2}$ even, and the $\psi^{M / 2, M}$ is also $Z_{2}$ even when $M$ is even.

Similarly, we use the same basis for the $X^{j, M}$, i.e.,

$$
X_{ \pm}^{j, M}=\frac{1}{\sqrt{2}}\left(X^{j, M}(z) \pm X^{M-j, M}(z)\right),
$$

except $j=0, M / 2$.

When $M=4$, only $X_{-}^{1,4}$ is $Z_{2}$ odd, and it is a singlet under the modular symmetry. Now, we study the other $Z_{2}$ even elements, $X^{0,4}, X_{+}^{1,4}$, and $X^{2,4}$. On $\left(X^{0,4}, X_{+}^{1,4}, X^{2,4}\right)^{T}$, $S$ and $T$ are represented by

$$
\begin{aligned}
& \rho(S)=\frac{\sqrt{i}}{2}\left(\begin{array}{ccc}
1 & \sqrt{2} & 1 \\
\sqrt{2} & 0 & -\sqrt{2} \\
1 & -\sqrt{2} & 1
\end{array}\right), \\
& \rho(T)=\left(\begin{array}{ccc}
1 & 0 & 0 \\
0 & e^{\pi i / 4} & 0 \\
0 & 0 & -1
\end{array}\right) .
\end{aligned}
$$

Here, let us study quartic tensor products,

$$
\left(X^{0,4}\right)^{\ell}\left(X_{+}^{1,4}\right)^{m}\left(X^{2,4}\right)^{n}
$$


with $\ell+m+n=4$. In total, there are fifteen elements. They provide us a reducible representation of $S$ and $T$. Thus, we decompose them into irreducible representations. The simplest one is the singlet $Z_{1}$, which is given by

$Z_{1}=\frac{2 \sqrt{2}}{\sqrt{3}}\left(\left(X_{+}^{1,4}\right)^{4}-2\left(\left(X^{0,4}\right)^{3} X^{2,4}+X^{0,4}\left(X^{2,4}\right)^{3}\right)\right)$.

On this singlet, $S$ and $T$ are represented by

$$
\rho(S)=-1, \quad \rho(T)=-1 .
$$

The next simplest irreducible representation is the doublet, $\left(Z_{2}, Z_{3}\right)$, which are given by

$$
\begin{aligned}
& Z_{2}=\left(X^{0,4}\right)^{4}+\left(X^{2,4}\right)^{4}+6\left(X^{0,4}\right)^{2}\left(X^{2,4}\right)^{2}, \\
& Z_{3}=\frac{4}{\sqrt{3}}\left(\left(X_{+}^{1,4}\right)^{4}+\left(X^{0,4}\right)^{3} X^{2,4}+X^{0,4}\left(X^{2,4}\right)^{3}\right) .
\end{aligned}
$$

On $\left(Z_{2}, Z_{3}\right)^{T}, S$ and $T$ are represented by the same $\rho(S)$ and $\rho(T)$ as Eq. (30). Thus, this is the $S_{3}$ doublet. Similarly, there is the triplet $\left(Z_{4}, Z_{5} \cdot Z_{6}\right)$, which are written by

$$
\begin{aligned}
& Z_{4}=\left(X^{0,4}\right)^{4}+\left(X^{2,4}\right)^{4}-2\left(X^{0,4}\right)^{2}\left(X^{2,4}\right)^{2}, \\
& Z_{5}=2 \sqrt{2}\left(X_{+}^{1,4}\right)^{2}\left(\left(X^{0,4}\right)^{2}+\left(X^{2,4}\right)^{2}\right), \\
& Z_{6}=4 \sqrt{2} X^{0,4}\left(X_{+}^{1,4}\right)^{2} X^{2,4} .
\end{aligned}
$$

On $\left(Z_{4}, Z_{5}, Z_{6}\right)^{T}, S$ and $T$ are represented by the same $\rho(S)$ and $\rho(T)$ as Eq. (32). Thus, this is the $S_{4}$ triplet.

At this stage, nine elements remain. Among nine elements, the $\left(Z_{7}, Z_{8}, Z_{9}\right)$ are a triplet, where

$$
\begin{aligned}
& Z_{7}=\left(X_{+}^{1,4}\right)^{2}\left(\left(X^{0,4}\right)^{2}-\left(X^{2,4}\right)^{2}\right), \\
& Z_{8}=X^{0,4} X_{+}^{1,4}\left(\left(X^{0,4}\right)^{2}-\left(X^{2,4}\right)^{2}\right), \\
& Z_{9}=-X^{2,4} X_{+}^{1,4}\left(\left(X^{0,4}\right)^{2}-\left(X^{2,4}\right)^{2}\right) .
\end{aligned}
$$

On this triplet $\left(Z_{7}, Z_{8}, Z_{9}\right)^{T}, S$ and $T$ are represented by

$$
\begin{aligned}
& \rho(S)=-\frac{1}{2}\left(\begin{array}{ccc}
0 & \sqrt{2} & \sqrt{2} \\
\sqrt{2} & 1 & -1 \\
\sqrt{2} & -1 & 1
\end{array}\right), \\
& \rho(T)=\left(\begin{array}{ccc}
i & 0 & 0 \\
0 & e^{\pi i / 4} & 0 \\
0 & 0 & e^{-\pi i / 4}
\end{array}\right) .
\end{aligned}
$$

The other 6 elements correspond to an irreducible representation, $\left(Z_{10}, Z_{11}, Z_{12}, Z_{13}, Z_{14}, Z_{15}\right)$, where

$$
\begin{aligned}
& Z_{10}=\left(X^{0,4}\right)^{4}-\left(X^{2,4}\right)^{4}, \\
& Z_{11}=\sqrt{2} X^{0,4} X_{+}^{1,4}\left(\left(X^{0,4}\right)^{2}+3\left(X^{2,4}\right)^{2}\right), \\
& Z_{12}=\sqrt{2} X^{2,4} X_{+}^{1,4}\left(3\left(X^{0,4}\right)^{2}+\left(X^{2,4}\right)^{2}\right) . \\
& Z_{13}=2 X^{0,4} X^{2,4}\left(X^{0,4}\right)^{2}-\left(X^{2,4}\right)^{2}, \\
& Z_{14}=2 \sqrt{2} X^{0,4}\left(X_{+}^{1,4}\right)^{3}, \\
& Z_{15}=2 \sqrt{2} X^{2,4}\left(X_{+}^{1,4}\right)^{3} .
\end{aligned}
$$

On them, $S$ is represented by

$$
\rho(S)=-\frac{1}{2}\left(\begin{array}{cccccc}
0 & 1 & 1 & 0 & 1 & 1 \\
1 & 0 & 0 & 1 & 1 & -1 \\
1 & 0 & 0 & 1 & -1 & 1 \\
0 & 1 & 1 & 0 & -1 & -1 \\
1 & 1 & -1 & -1 & 0 & 0 \\
1 & -1 & 1 & -1 & 0 & 0
\end{array}\right),
$$

and $T$ is represented by

$$
\rho(T)=\operatorname{diag}\left(1, e^{\pi i / 4},-e^{\pi i / 4},-1, e^{3 \pi i / 4},-e^{3 \pi i / 4}\right) .
$$

Both representations on $\left(Z_{7}, Z_{8}, Z_{9}\right)$ and $\left(Z_{10}, Z_{11}, Z_{12}\right.$, $\left.Z_{13}, Z_{14}, Z_{15}\right)$ satisfy

$$
\begin{array}{ll}
\rho\left(S^{2}\right)=\mathbb{I}_{N \times N}, & \rho\left((S T)^{3}\right)=\mathbb{I}_{N \times N}, \\
\rho\left(T^{8}\right)=\mathbb{I}_{N \times N}, & \rho\left(\left(S T^{-1} S T\right)^{3}\right)=\mathbb{I}_{N \times N},
\end{array}
$$

for $N=3$ and 6 , respectively. This is the $\Delta(96)$ algebra. In particular, $\left(Z_{7}, Z_{8}, Z_{9}\right)$ correspond to the $\Delta(96)$ triplet.

\section{Larger $M$}

Similarly, we can study the case with larger $M$. Quartic tensor products of $X^{j, M}$ would be modular forms of finite modular subgroups. We have obtained modular forms corresponding to $S_{3} \simeq \Delta(6), S_{4} \simeq \Delta(24), \Delta(96)$. These are the $\Delta\left(6 N^{2}\right)$ series. Thus, we may obtain modular forms of $\Delta\left(6 N^{2}\right)$ with larger $N$ for larger $M$.

For example, we consider the case with $M=8$. Then, we use the $Z_{2}$ even orbifold basis, i.e., $\left(X^{0,8}, X_{+}^{1,8}, X_{+}^{2,8}\right.$, $\left.X_{+}^{3,8}, X^{4,8}\right)$. On them, $S$ is represented by

$\rho(S)=\sqrt{\frac{i}{8}}\left(\begin{array}{ccccc}1 & \sqrt{2} & \sqrt{2} & \sqrt{2} & 1 \\ \sqrt{2} & \sqrt{2} & 0 & -\sqrt{2} & -\sqrt{2} \\ \sqrt{2} & 0 & -2 & 0 & \sqrt{2} \\ \sqrt{2} & -\sqrt{2} & 0 & \sqrt{2} & -\sqrt{2} \\ 1 & -\sqrt{2} & \sqrt{2} & -\sqrt{2} & 1\end{array}\right)$,

and $T$ is represented by 


$$
\rho(T)=\operatorname{diag}\left(1, e^{\pi i / 8}, e^{\pi i / 2}, e^{9 \pi i / 8}, 1\right) .
$$

Then, we consider quartic tensor products. For instance, there is a triplet, $\left(Z_{1}, Z_{2}, Z_{3}\right)$, where

$$
\begin{aligned}
Z_{1}= & 2\left(X^{0,8}\left(X_{+}^{1,8}\right)^{2} X_{+}^{2,8}+\left(X^{1,8}\right)^{2} X_{+}^{2,8} X^{4,8}\right. \\
& \left.-X^{0,8} X_{+}^{2,8}\left(X_{+}^{3,8}\right)^{2}-X_{+}^{2,8}\left(X_{+}^{3,8}\right)^{2} X^{4,8}\right), \\
Z_{2}= & \left(X^{0,8}\right)^{3} X_{+}^{1,8}-X_{+}^{1,8}\left(X^{4,8}\right)^{3}+\left(X^{0,8}\right)^{2} X_{+}^{1,8} X^{4,8} \\
& -X^{0,8} X_{+}^{1,8}\left(X^{4,8}\right)^{2}+2 X^{0,8}\left(X_{+}^{2,8}\right)^{2} X^{3,8} \\
& -2\left(X_{+}^{2,8}\right)^{2} X_{+}^{3,8} X^{4,8}, \\
Z_{3}= & -\left(\left(X^{0,8}\right)^{3} X_{+}^{3,8}-X_{+}^{3,8}\left(X^{4,8}\right)^{3}+\left(X^{0,8}\right)^{2} X_{+}^{3,8} X^{4,8}\right. \\
& -X^{0,8} X_{+}^{3,8}\left(X^{4,8}\right)^{2}+2 X^{0,8}\left(X_{+}^{2,8}\right)^{2} X^{1,8} \\
& \left.-2\left(X_{+}^{2,8}\right)^{2} X_{+}^{1,8} X^{4,8}\right) .
\end{aligned}
$$

On them, $S$ and $T$ are represented by

$$
\begin{aligned}
& \rho(S)=\frac{1}{2}\left(\begin{array}{ccc}
0 & \sqrt{2} & \sqrt{2} \\
\sqrt{2} & -1 & 1 \\
\sqrt{2} & 1 & -1
\end{array}\right), \\
& \rho(T)=\left(\begin{array}{ccc}
e^{6 \pi i / 8} & 0 & 0 \\
0 & e^{9 \pi i / 8} & 0 \\
0 & 0 & e^{\pi i / 8}
\end{array}\right) .
\end{aligned}
$$

They correspond to the $\Delta(384)$ triplet. Similarly, we can discuss the case with larger $M$.

\section{CONCLUSION}

We have studied modular transformation of holomorphic Yukawa couplings in magnetized D-brane models. Their products correspond to nontrivial representations of finite modular subgroups. Explicitly, we have constructed modular forms of weight 2 for the $S_{3}$ doublet, the $S_{4}$ triplet, the $\Delta(96)$ triplet and sextet, and the $\Delta(384)$ triplet. Similarly, these products with larger $M$ would give us representations for $\Gamma_{2 M}$.

We have set vanishing Wilson lines. In our discussion, the $Z_{2}$ orbifold basis is important. The orbifold with magnetic flux allows discrete Wilson lines [33]. It would be interesting to extend our analysis to magnetized D-brane models with discrete Wilson lines.

Our results would be useful to study model building for the quark and lepton masses and mixing angles by extending analyses in Refs. [22-27]. Although our purpose is to construct modular forms of weight 2 for finite modular subgroups, it is also a challenging issue to study string compactifications, where our modular forms are realized as Yukawa couplings.

\section{ACKNOWLEDGMENTS}

T. K. was is supported in part by MEXT KAKENHI Grant No. JP17H05395.
[1] G. Altarelli and F. Feruglio, Rev. Mod. Phys. 82, 2701 (2010).

[2] H. Ishimori, T. Kobayashi, H. Ohki, Y. Shimizu, H. Okada, and M. Tanimoto, Prog. Theor. Phys. Suppl. 183, 1 (2010); Lect. Notes Phys. 858, 1 (2012).

[3] S. F. King and C. Luhn, Rep. Prog. Phys. 76, 056201 (2013).

[4] T. Kobayashi, H. P. Nilles, F. Ploger, S. Raby, and M. Ratz, Nucl. Phys. B768, 135 (2007).

[5] T. Kobayashi, S. Raby, and R. J. Zhang, Nucl. Phys. B704, 3 (2005).

[6] P. Ko, T. Kobayashi, J. h. Park, and S. Raby, Phys. Rev. D 76, 035005 (2007); 76, 059901(E) (2007).

[7] Y. Olguin-Trejo, R. Perez-Martinez, and S. Ramos-Sanchez, Phys. Rev. D 98, 106020 (2018).

[8] H. P. Nilles, M. Ratz, A. Trautner, and P. K. S. Vaudrevange, Phys. Lett. B 786, 283 (2018).

[9] F. Beye, T. Kobayashi, and S. Kuwakino, Phys. Lett. B 736, 433 (2014).

[10] H. Abe, K. S. Choi, T. Kobayashi, and H. Ohki, Nucl. Phys. B820, 317 (2009).
[11] H. Abe, K. S. Choi, T. Kobayashi, and H. Ohki, Phys. Rev. D 80, 126006 (2009); 81, 126003 (2010).

[12] M. Berasaluce-Gonzalez, P. G. Camara, F. Marchesano, D. Regalado, and A. M. Uranga, J. High Energy Phys. 09 (2012) 059.

[13] F. Marchesano, D. Regalado, and L. Vazquez-Mercado, J. High Energy Phys. 09 (2013) 028.

[14] H. Abe, T. Kobayashi, H. Ohki, K. Sumita, and Y. Tatsuta, J. High Energy Phys. 06 (2014) 017.

[15] T. Kobayashi and S. Nagamoto, Phys. Rev. D 96, 096011 (2017).

[16] T. Kobayashi, S. Nagamoto, S. Takada, S. Tamba, and T. H. Tatsuishi, Phys. Rev. D 97, 116002 (2018).

[17] D. Cremades, L. E. Ibanez, and F. Marchesano, J. High Energy Phys. 05 (2004) 079.

[18] J. Lauer, J. Mas, and H. P. Nilles, Phys. Lett. B 226, 251 (1989); Nucl. Phys. B351, 353 (1991).

[19] W. Lerche, D. Lust, and N. P. Warner, Phys. Lett. B 231, 417 (1989).

[20] S. Ferrara, D. Lust, and S. Theisen, Phys. Lett. B 233, 147 (1989). 
[21] R. de Adelhart Toorop, F. Feruglio, and C. Hagedorn, Nucl. Phys. B858, 437 (2012).

[22] F. Feruglio, arXiv:1706.08749.

[23] T. Kobayashi, K. Tanaka, and T. H. Tatsuishi, Phys. Rev. D 98, 016004 (2018).

[24] J. T. Penedo and S. T. Petcov, Nucl. Phys. B939, 292 (2019).

[25] J. C. Criado and F. Feruglio, SciPost Phys. 5, 042 (2018).

[26] T. Kobayashi, N. Omoto, Y. Shimizu, K. Takagi, M. Tanimoto, and T.H. Tatsuishi, J. High Energy Phys. 11 (2018) 196.

[27] P. P. Novichkov, J. T. Penedo, S. T. Petcov, and A. V. Titov, arXiv:1811.04933.
[28] R. C. Gunning, Lectures on Modular Forms (Princeton University Press, Princeton, NJ, 1962).

[29] B. Schoeneberg, Elliptic Modular Functions (SpringerVerlag, 1974).

[30] N. Koblitz, Introduction to Elliptic Curves and Modular Forms (Springer-Verlag, 1984).

[31] H. Abe, K. S. Choi, T. Kobayashi, and H. Ohki, J. High Energy Phys. 06 (2009) 080.

[32] H. Abe, T. Kobayashi, and H. Ohki, J. High Energy Phys. 09 (2008) 043.

[33] T. H. Abe, Y. Fujimoto, T. Kobayashi, T. Miura, K. Nishiwaki, and M. Sakamoto, J. High Energy Phys. 01 (2014) 065; Nucl. Phys. B890, 442 (2014). 